\title{
Early Evaluation of PSA Response in Metastatic Prostate Cancer Treated with Abiraterone
}

\author{
Ana Santafé-Jiménez, Virginia Morillo-Macías, Angel Sánchez-Iglesias, \\ Encarnación Fernández-Camacho, Carlos Ferrer-Albiach \\ Consorcio Hospitalario Provincial de Castellón, Castellón de la Plana, Spain \\ Email: a.santafe93mail.com
}

How to cite this paper: Santafé-Jiménez, A., Morillo-Macías, V., Sánchez-Iglesias, A., Fernández-Camacho, E. and FerrerAlbiach, C. (2021) Early Evaluation of PSA Response in Metastatic Prostate Cancer Treated with Abiraterone. Open Journal of Urology, 11, 251-263.

https://doi.org/10.4236/oju.2021.117023

Received: June 13, 2021

Accepted: July 17, 2021

Published: July 20, 2021

Copyright $\odot 2021$ by author(s) and Scientific Research Publishing Inc. This work is licensed under the Creative Commons Attribution International License (CC BY 4.0).

http://creativecommons.org/licenses/by/4.0/

\begin{abstract}
Background: According to the main prostate cancer guidelines, the response to treatment with abiraterone plus prednisone $(\mathrm{AA}+\mathrm{P})$ must be evaluated by assessing prostate-specific antigen (PSA) levels at 12 weeks. Recent studies have shown that early PSA decline, at 4 weeks, maybe a surrogate marker for survival. The objective of this work was to analyze if a decline in PSA at 4 weeks correlates with a better outcome in terms of OS (overall survival) and PFS (progression-free survival). Methods: We evaluated 168 patients (with a median age of 71 years) with prostate cancer who had started $\mathrm{AA}+\mathrm{P}$ treatment between February 2012 and July 2019. Patients were divided into three different groups according to the decline of PSA ( $\geq 30 \%, \geq 50 \%$, and $\geq 90 \%)$ at 4,8 , and 12 weeks. Statistical survival analysis was performed using the Kaplan-Meier method. Results: After a follow-up of 69 months, a PSA decline $\geq$ $30 \%$ at 4 weeks was associated with longer median OS times (28 vs. 18 months; $p=0.027$ ). A decline in PSA by $\geq 50 \%$ was also associated with increased median OS times (36 vs. $21 ; p=0.003$ ). Cox univariable analysis indicated that a decrease in PSA (both by $\geq 30 \%$ and $\geq 50$ ) were predictive of OS at 4 weeks $(\mathrm{PSA} \geq 30 \%: \mathrm{HR}=1.568,95 \% \mathrm{CI}[1.041,2.360], p=0.031$; $\mathrm{PSA} \geq 50 \%: \mathrm{HR}=$ $1.901,95 \%$ CI $[1.222,2.956], p=0.004)$; although multivariable analysis did not confirm these results. The prior administration of chemotherapy was an independent risk factor for death $(\mathrm{HR}=2.511 ; p<0.001)$ and progression ( $\mathrm{HR}=3.238 ; p<0.001)$, probably because of different factors. Conclusion: A decrease in PSA by $\geq 30 \%$ or $\geq 50 \%$ at 4 weeks after starting treatment with $\mathrm{AA}+\mathrm{P}$ correlated with longer PFS and OS, and provides clinically meaningful information guiding the physicians towards a personalized treatment.
\end{abstract}

\section{Keywords}

Prostate Cancer, Abiraterone, Prostate-Specific Antigen Decline, Survival 


\section{Introduction}

Abiraterone acetate (AA) is a second-generation antiandrogen; it is a selective inhibitor of the enzyme 17 alpha-hydroxylase/C1,20-lyase (CYP17) [1]. Following the excellent survival results obtained in pivotal trials [2] [3] [4], the administration of abiraterone together with prednisone $(\mathrm{AA}+\mathrm{P})$ has been approved by health authorities as a treatment for pre-chemotherapy castration-resistant metastatic prostate cancer (pre-CT mCRPC), post-chemotherapy metastatic prostate cancer (post-CT mCRPC), and hormone-sensitive metastatic prostate cancer (mHSPC).

The timely identification of non-responders from among patients treated with $\mathrm{AA}+\mathrm{P}$ is crucial so that they can potentially benefit from an early change in their treatment regimen. Except for PSA (prostate-specific antigen), no sensitive and accessible predictive biomarkers for the evaluation of $\mathrm{AA}+\mathrm{P}$ treatment are yet available. However, the evaluation of PSA is also associated with a series of limitations such as the flare effect.

The latest recommendations from the Prostate Cancer Working Group 3 (PCWG3) [5] indicate that the first PSA measurement after the start of treatment should not be carried out before 12 weeks in order to avoid potential biases caused by these aforementioned phenomena. Thus, data on the kinetics of PSA after the first 4 weeks of treatment are limited because none of the pivotal trials performed to date evaluated the decrease in PSA before 3 months. Despite this, some recent publications have evaluated PSA at 4 weeks with promising results, suggesting that PSA could act as a surrogate marker for survival.

Until 2019, these studies had focused solely on mCRPC patients and found that a decrease in PSA by $\geq 30 \%$ or $\geq 50 \%$ was related with increased survival [6] [7] [8] [9] [10]. Recently, two studies that exclusively analyzed mHSPC patients added the variable of a decrease in PSA by $\geq 90 \%$ and found similar data [11] [12]. These studies suggested that professionals should consider early changes in PSA because this marker can provide information on the efficacy of treatments, the need to interrupt therapies, and their associated prognosis.

In this present work, our objective was to analyze whether a decrease in PSA greater than $30 \%, 50 \%$, and/or $90 \%$ at 4 weeks in our patient groups correlated with an improvement in OS and PFS. We wanted to ascertain whether this factor could serve as an early predictive marker of a good response to AA+P therapy. Finally, we also analyzed these data at 8 and 12 weeks to compare the results with the earliest response at 4 weeks.

\section{Material and Methods}

This is a retrospective, multi-center study carried out at two centers attached to our health department (Consorcio Hospitalario Provincial de Castellón, Hospital Universitario General de Castellón). This work included 172 patients diagnosed with prostate adenocarcinoma who had started treatment with $\mathrm{AA}+\mathrm{P}$ between February 2012 and July 2019. After more thorough evaluation, 4 patients were 
excluded because insufficient data were available for them. We collected demographic and biometric data including information regarding their personal history, the histological characteristics of the tumor, staging (TNM 8th edition [13]), initial treatment, and dates of relapses and resistance to castration, if any. All these variables were selected based on previously conducted pivotal studies and were collected using an SPSS dataset.

Since this was a retrospective study we did not have an ethical protocol, but patients received the informed consent documents for treatment and we complied with the data protection law.

Patients were divided into 3 groups: patients with metastatic castration-resistant prostate cancer who had previously received docetaxel-based chemotherapy (postCT mCRPC), patients with metastatic castration-resistant prostate cancer with no previous chemotherapy (pre-CT $\mathrm{mCRPC}$ ) and patients with metastatic hormone-sensitive prostate cancer (mHSPC). The PCWG3 castration-resistant definition was used.

PSA figures were collected at 4, 8, and 12 weeks after the initiation of AA+P, and then every 6 months thereafter. The patients were classified considering the percentage of the decrease in PSA registered at each time point. Within each of the periods, the patients were subdivided into three groups according to whether there had been a decrease in PSA greater than or equal to $90 \%, 50 \%$, and $30 \%$, respectively.

In addition, analytical data were collected before and during the $\mathrm{AA}+\mathrm{P}$ treatment. These included lactate dehydrogenase (LDH), alkaline phosphatase (AP), liver enzymes, potassium, cholesterol, triglycerides, and the neutrophil to lymphocyte ratio (neu/lym index). The time to the PSA nadir was defined as the time from the start of $\mathrm{AA}+\mathrm{P}$ treatment to the date with the lowest PSA figure, quantified in months. This variable was divided into three periods: $<6$ months, between 6 and 12 months, and $>12$ months.

All the calculations were carried out using SPSS software (version24, IBM Corp., Armonk, NY). OS was defined as the time from the start of treatment to the date of death or the last update on the patient's condition. PFS was defined as the time from the start of treatment to the date of radiological progression or clinical deterioration. Both OS and PFS were calculated with the Kaplan-Meier method, and the differences between the curves were analyzed with the Log-Rank test, using $p<0.05$ as a reference value.

A univariate COX model was used to evaluate the effect of a decrease in PSA by $\geq 30 \%, \geq 50 \%$, or $\geq 90 \%$ at 4,8 , and 12 weeks in terms of OS and PFS. In addition, multivariate analysis was used to analyze these variables together with other factors such as LDH, AP, neu/lym index, previous administration of chemotherapy, Gleason score at diagnosis, presence of cardiovascular factors (hypertension, diabetes mellitus, dyslipidemia), and others. Finally, Spearman correlations were used to analyze the relationship between PSA levels and their decline at 4 , 8 , and 12 weeks. 


\section{Results}

Of the 168 patients, $49.4 \%$ were in the pre-CT mCRPC group, $36.9 \%$ were identified as post-CT mCRPC, and the rest (13.7\%) had mCPHS. Table 1 summarizes the baseline characteristics of the overall sample, and Tables 2-4 show the baseline characteristics of these patients according to these afore mentioned subdivisions. In the mHSPC group all patients debuted as metastatic and most of them received AA as first treatment following the LATITUDE protocol criteria. On the other hand, in the MCRPC group most patients were not metastatic at the time of disease diagnosis but were included in the locally advanced stage. In

Table 1. Baseline characteristics of overall sample.

\begin{tabular}{cccccc}
\hline \multicolumn{5}{c}{ OVERALL SAMPLE $(\boldsymbol{n}=168)$} \\
\hline \multicolumn{5}{c}{ Average age: 70.60 years (range = 47 - 92) } \\
\hline DM & Yes: $16.7 \%$ & No: $83.3 \%$ & & \\
PAH & Yes: $53.6 \%$ & No: $46.4 \%$ & & & \\
DLP & Yes: $37 \%$ & No $63 \%$ & & & \\
Statins & Yes: $30.3 \%$ & No: $69.7 \%$ & & G10: $5.4 \%$ \\
Gleason & G6: $13.6 \%$ & G7: $29.9 \%$ & G8: $26.5 \%$ & G9: $24.5 \%$ & T10 \\
T & T1: $0.9 \%$ & T2: $22.3 \%$ & T3: $55.4 \%$ & T4: $21.4 \%$ & \\
N & N0: $60.7 \%$ & N1: $29.9 \%$ & N2: $5.6 \%$ & N3: $3.7 \%$ & \\
M & M0: $49.1 \%$ & M1a: $5 \%$ & M1b: $46 \%$ & & \\
Risk & Low: $2.4 \%$ & Medium: $7.1 \%$ & High: $81.5 \%$ & & \\
1st Treatment & RT: $25.5 \%$ & PRL: $14.5 \%$ & HT: $46.1 \%$ & AA: $10.3 \%$ & CT: $2.4 \%$ \\
\hline
\end{tabular}

Abbreviations: DM, diabetes mellitus; PAH, pulmonary arterial hypertension; DLP, dyslipidemia; RT, radiotherapy; PRL, prostatectomy; HT, hormone therapy; AA, abiraterone acetate; $\mathrm{CT}$, chemotherapy.

Table 2. Baseline characteristics of mHSPC sample.

\begin{tabular}{|c|c|c|c|c|c|}
\hline \multicolumn{6}{|c|}{$\operatorname{mHSPC}(n=23)$} \\
\hline \multicolumn{6}{|c|}{ Average age: 68.70 years (Range $=47-81$ ) } \\
\hline $\mathrm{DM}$ & Yes: $17.4 \%$ & No: $82.6 \%$ & & & \\
\hline PAH & Yes: $43.5 \%$ & No: $56.5 \%$ & & & \\
\hline DLP & Yes: $35 \%$ & No $65 \%$ & & & \\
\hline Statins & Yes: $30 \%$ & No: $70 \%$ & & & \\
\hline Gleason & G6: $0 \%$ & G7: $4.3 \%$ & G8: $47.8 \%$ & G9: $43.5 \%$ & G10: $4.3 \%$ \\
\hline $\mathrm{T}$ & $\mathrm{T} 1: 0 \%$ & T2: $11.1 \%$ & T3: $44.4 \%$ & $\mathrm{~T} 4: 44.4 \%$ & \\
\hline $\mathbf{N}$ & N0: $10.5 \%$ & $\mathrm{~N} 1: 47.4 \%$ & $\mathrm{~N} 2: 31.6 \%$ & N3: $10.5 \%$ & \\
\hline $\mathbf{M}$ & M0: $0 \%$ & M1a: 0\% & M1b: $100 \%$ & & \\
\hline Risk & Low: $0 \%$ & Medium: 0\% & High: $100 \%$ & & \\
\hline 1st Treatment & RT: $0 \%$ & PRL: $0 \%$ & HT: $19 \%$ & AA: $81 \%$ & CT: $0 \%$ \\
\hline
\end{tabular}

Abbreviations: DM, diabetes mellitus; PAH, pulmonary arterial hypertension; DLP, dyslipidemia; RT, radiotherapy; PRL, prostatectomy; HT, hormone therapy; AA, abiraterone acetate; CT, chemotherapy. 
Table 3. Baseline characteristics of pre-CT mCPRC sample.

\begin{tabular}{cccccc}
\hline \multicolumn{5}{c}{ pre-CT mCRPC $(\boldsymbol{n}=\mathbf{8 3})$} & \\
\hline \multicolumn{5}{c}{ Average age: 73.48 years (Range = 49 - 89) } \\
\hline DM & Yes: $19.3 \%$ & No: $80.7 \%$ & & \\
PAH & Yes: $60.2 \%$ & No: $39.8 \%$ & & & \\
DLP & Yes: $47 \%$ & No $53 \%$ & & & \\
Statins & Yes: $37.3 \%$ & No: $62.7 \%$ & & G9: $15.3 \%$ & G10: $4.2 \%$ \\
Gleason & G6: $20.8 \%$ & G7: $33.3 \%$ & G8: $26.4 \%$ & T4: $\% .1 \%$ & \\
T & T1: $1.8 \%$ & T2: $23.6 \%$ & T3: $65.5 \%$ & N3: $4 \%$ & \\
N & N0: $72 \%$ & N1: $24 \%$ & N2: $0 \%$ & & \\
M & M0: $68.4 \%$ & M1a: $7.6 \%$ & M1b: $24.1 \%$ & & \\
Risk & Low: $3.6 \%$ & Medium: $9.6 \%$ & High: $77.1 \%$ & & CT: $0 \%$ \\
\hline 1st Treatment & RT: $36.6 \%$ & PRL: $20.7 \%$ & HT: $42.7 \%$ & AA: $0 \%$ & \\
\hline
\end{tabular}

Abbreviations: DM, diabetes mellitus; PAH, pulmonary arterial hypertension; DLP, dyslipidemia; RT, radiotherapy; PRL, prostatectomy; HT, hormone therapy; AA, abiraterone acetate; CT, chemotherapy.

Table 4. Baseline characteristics of post-CT mCPRC sample.

\begin{tabular}{cccccc}
\hline \multicolumn{5}{c}{ post-CT mCPRC $(n=62)$} & \\
\hline \multicolumn{5}{c}{ Average age: 67.45 years (Range = 50 - 92) } \\
\hline DM & Yes: $12.9 \%$ & No: $87.1 \%$ & & & \\
AH & Yes: $48.4 \%$ & No: $51.6 \%$ & & & \\
DLP & Yes: $24.2 \%$ & No: $75.8 \%$ & & & \\
Statins & Yes: $21 \%$ & No: $79 \%$ & & G9: $28.8 \%$ & G10: $7.7 \%$ \\
Gleason & G6: $9.6 \%$ & G7: $36.5 \%$ & G8: $17.3 \%$ & T4: $28.2 \%$ & \\
T & T1: $0 \%$ & T2: $25.6 \%$ & T3: $46.2 \%$ & N3: $0 \%$ & \\
N & N0: $71.1 \%$ & N1: $28.9 \%$ & N2: $0 \%$ & & \\
M & M0: $42.4 \%$ & M1a: $3.4 \%$ & M1b: $54.2 \%$ & & \\
Risk & Low: $1.6 \%$ & Medium: $6.5 \%$ & High: $80.6 \%$ & & CT: $6.5 \%$ \\
\hline 1st Treatment & RT: $19.4 \%$ & RP: $12.9 \%$ & HT: $59.7 \%$ & AA: $0 \%$ & \\
\hline
\end{tabular}

Abbreviations: DM, diabetes mellitus; PAH, pulmonary arterial hypertension; DLP, dyslipidemia; RT, radiotherapy; PRL, prostatectomy; HT, hormone therapy; AA, abiraterone acetate; $\mathrm{CT}$, chemotherapy.

the pre-CT mCRPC, more than $60 \%$ were staged as T3 and almost $80 \%$ belonged to the high-risk classification, according to NCCN guidelines. In the latter group (post-CT mCRPC) had a higher proportion of patients staged as T4 and it is noteworthy that approximately $7 \%$ of patients received CT as first treatment.

At 4 weeks, $52 \%$ of all the patients had presented a decrease in PSA by $\geq 30 \%$, $39 \%$ had exceeded a $50 \%$ decrease, and the decrease in PSA exceeded $90 \%$ in $10.1 \%$ of the total sample. As might be expected, the proportions between these data at 8 and 12 weeks were like those from 4 weeks, although the percentages were progressively higher (see Table 5). The median PSA nadir was $6.82 \mathrm{ng} / \mathrm{mL}$ 
Table 5. Percentage of patients according to PSA decline at 4, 8 and 12 weeks.

\begin{tabular}{ccccccc}
\hline PSA decrease & $\mathbf{3 0 \%}$ & $<\mathbf{3 0 \%}$ & $\mathbf{2 5 0 \%}$ & $<50 \%$ & $\geq 90$ & $<90 \%$ \\
\hline 4 weeks & $52.0 \%$ & $48.0 \%$ & $39.2 \%$ & $60.8 \%$ & $10.1 \%$ & $89.9 \%$ \\
8 weeks & $56.8 \%$ & $43.2 \%$ & $48.7 \%$ & $51.3 \%$ & $13.4 \%$ & $86.6 \%$ \\
12 weeks & $62.8 \%$ & $37.2 \%$ & $56.2 \%$ & $43.8 \%$ & $26.3 \%$ & $73.7 \%$ \\
\hline
\end{tabular}

Abbreviations: PSA, prostate-specific antigen.

(range $=0.00-1232$ ) and the mean time to the PSA nadir (tPSAn) was 7.69 months. A longer tPSAn was associated with better OS and PFS, as shown in Table 6.

In addition, at 4 weeks, patients who had shown a decrease in PSA by $\geq 30 \%$ had a better median OS (28 vs. 19 months; $p=0.027$ ) (Figure 1 ) and median PFS (17 vs. 6 months; $p<0.001$ ). Similarly, in the subgroup whose PSA had decreased by $\geq 50 \%$, we also observed significant differences in median OS ( 36 vs. 21 months; $p=0.003$ ) (Figure 2) and median PFS (20 vs. 8 months; $p<0.001$ ). Finally, a decrease in PSA by $\geq 90 \%$ at 4 weeks was related to an improvement in median PFS (31 vs. 11 months; $p=0.006$ ) (Figure 3); however, the relationship was not significant between this parameter and median OS, although it did follow the same trend ( 57 vs. 24 months; $p=0.063$ ). The results were similar at 8 and 12 weeks, with an increase in OS and PFS observed in patients whose PSA had decreased by $\geq 30 \%$ or $\geq 50 \%$. The decrease in PSA by $\geq 90 \%$ was only significantly related to an improvement in PFS, not in OS.

To clarify if there were any differences that could distort the results we performed an analysis by the three subgroups that include our sample, as shown in the following Tables 7-10: in the mCRPC group a decrease in PSA $\geq 50 \%$ and $\geq 90 \%$ was related with a significant better outcome in terms of OS and PFS. However, when analyzing the two subgroups inside this group (pre-CT mCRPC and post-CT mCRPC) we did not find any significant results. In the last group (mHSPC), we observed a relationship between a PSA decline $\geq 30 \%$ and an improvement in OS and PFS, but not with a PSA decrease $\geq 50 \%$ and $\geq 90 \%$. All these results are given as median survivals, except for the ones that refer to the PSA decrease $\geq 90 \%$ that are given as mean survival because this group of data did not achieve the fiftieth percentile.

The univariate analysis showed that, at 4 weeks, both decreases by $\geq 30 \%$ and $\geq 50 \%$ were related to increased OS (HR $=1.568,95 \%$ CI $[1.041,2.360], p=$ $0.031 ; \mathrm{HR}=1.901,95 \% \mathrm{CI}[1.222,2.956], p=0.004)$ and increased PFS (HR $=$ 2.036, 95\% CI [1.371, 3.025], $p<0.001$; HR $=2.236,95 \%$ CI [1.465, 3.412], $p<$ 0.001). However, we were unable to corroborate this result in the multivariate analysis (see Table 11). Nonetheless, it should also be noted that, in the overall sample, administration of chemotherapy prior to $\mathrm{AA}+\mathrm{P}$ was a risk factor which was associated with a reduced time to progression $(\mathrm{HR}=3.238 ; p<0.001)$ or death $(\mathrm{HR}=2.511 ; p<0.001)$. Moreover, this variable remained statistically significant in the multivariate analysis. 
Table 6. Relationship between time to PSA nadir and survival.

Time to PSA nadir Time to progression (months) $p$-value Time to death (months) $p$-value

$\begin{array}{lcccc}<6 \text { months } & 8.87 & 18.14 & \\ 4-6 \text { months } & 23.76 & <0.001 & 32.70 & <0.001 \\ >12 \text { months } & 44.06 & & 58.09 & \end{array}$

Abbreviations: PSA, prostate-specific antigen.

Table 7. Results of survival in m-CRPC group.

\begin{tabular}{cccc}
\hline & $<30 \%$ & $>30 \%$ & $p$ value \\
\hline Median PFS & 7 & 16 & 0.004 \\
Median OS & 21 & 26 & 0.83 \\
\hline Median PFS & $<50 \%$ & $>50 \%$ & $p$ value \\
Median OS & 8 & 18 & 0.001 \\
\hline Mean PFS & 21 & 36 & 0.009 \\
Mean OS & $<90 \%$ & $>90 \%$ & $p$ value \\
\hline
\end{tabular}

Abbreviations: OS, overall survival; PFS, progression-free survival.

Table 8. Results of survival in pre-CT m-CRPC group.

\begin{tabular}{cccc}
\hline & $<\mathbf{3 0 \%}$ & $>\mathbf{3 0 \%}$ & $p$ value \\
\hline Median PFS & 17 & 20 & 0.297 \\
Median OS & 29 & 40 & 0.793 \\
\hline Median PFS & $<50 \%$ & $>\mathbf{5 0 \%}$ & $\boldsymbol{p}$ value \\
Median OS & 16 & 29 & 0.055 \\
\hline Mean PFS & 28 & 45 & 0.104 \\
\hline Mean OS & $<90 \%$ & $>90 \%$ & $p$ value \\
\hline
\end{tabular}

Abbreviations: OS, overall survival; PFS, progression-free survival.

Table 9. Results of survival in post-CT m-CRPC group.

\begin{tabular}{cccc}
\hline & $<30 \%$ & $>30 \%$ & $p$ value \\
\hline Median PFS & 4 & 11 & 0.069 \\
Median OS & 13 & 24 & 0.269 \\
\hline Median PFS & $<50 \%$ & $>50 \%$ & $p$ value \\
Median OS & 4 & 11 & 0.079 \\
\hline Mean PFS & 13 & 25 & 0.130 \\
\hline
\end{tabular}

Abbreviations: OS, overall survival; PFS, progression-free survival. 


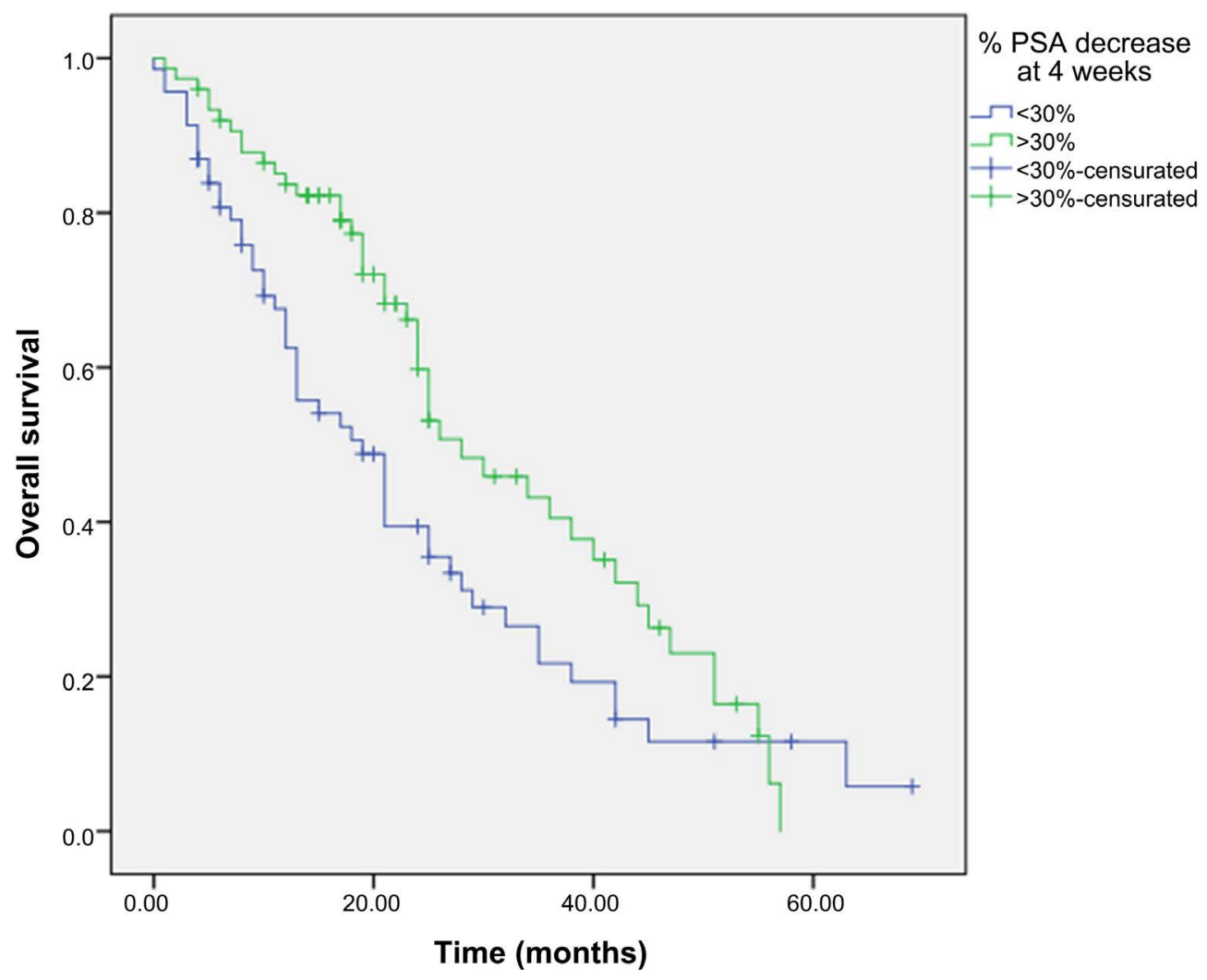

Figure 1. Overall survival curve as a function of the decrease in PSA (by $>30 \%$ and $<30 \%$ ).

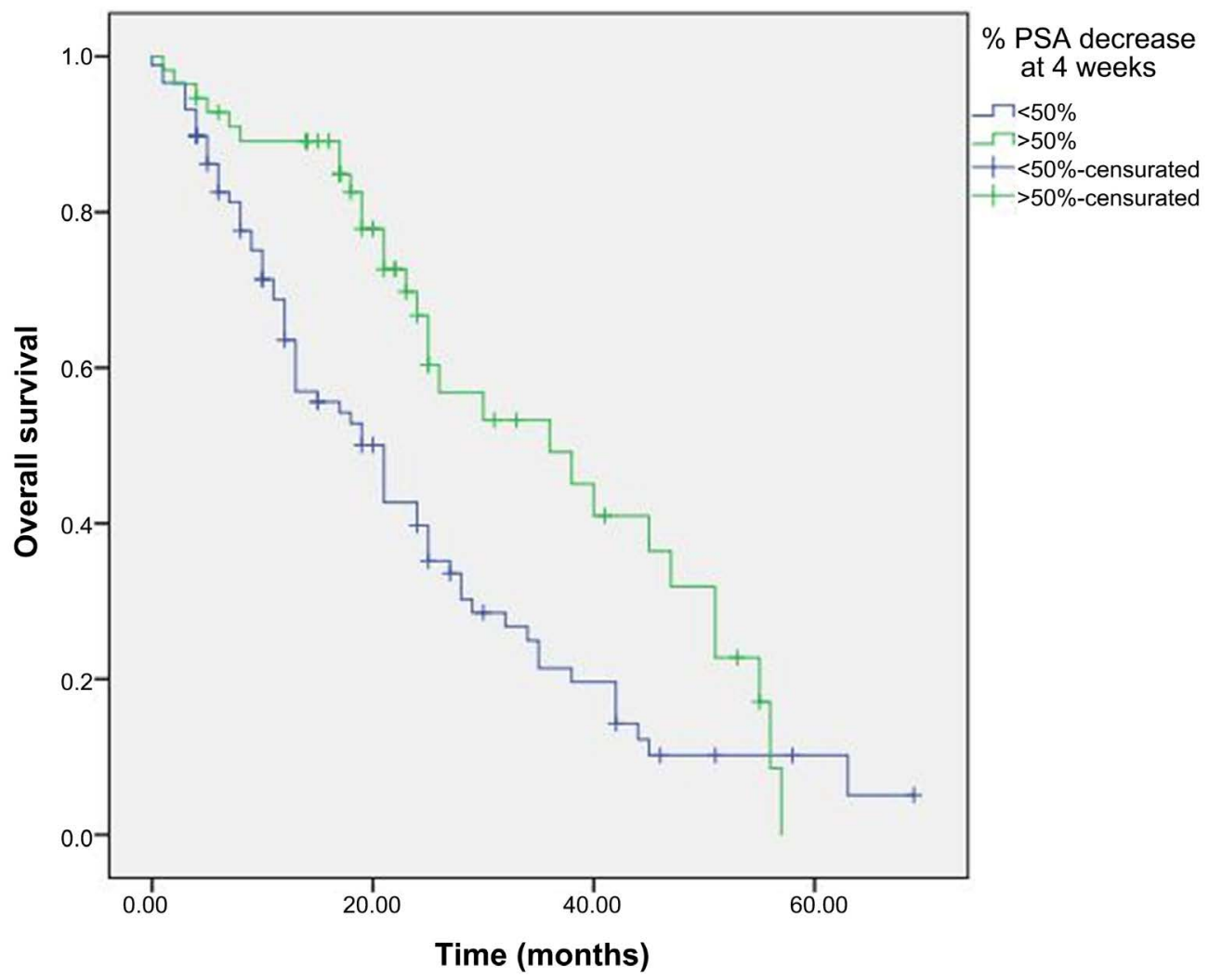

Figure 2. Overall survival curve as a function of the decrease in PSA (by $>50 \%$ and $<50 \%$ ). 


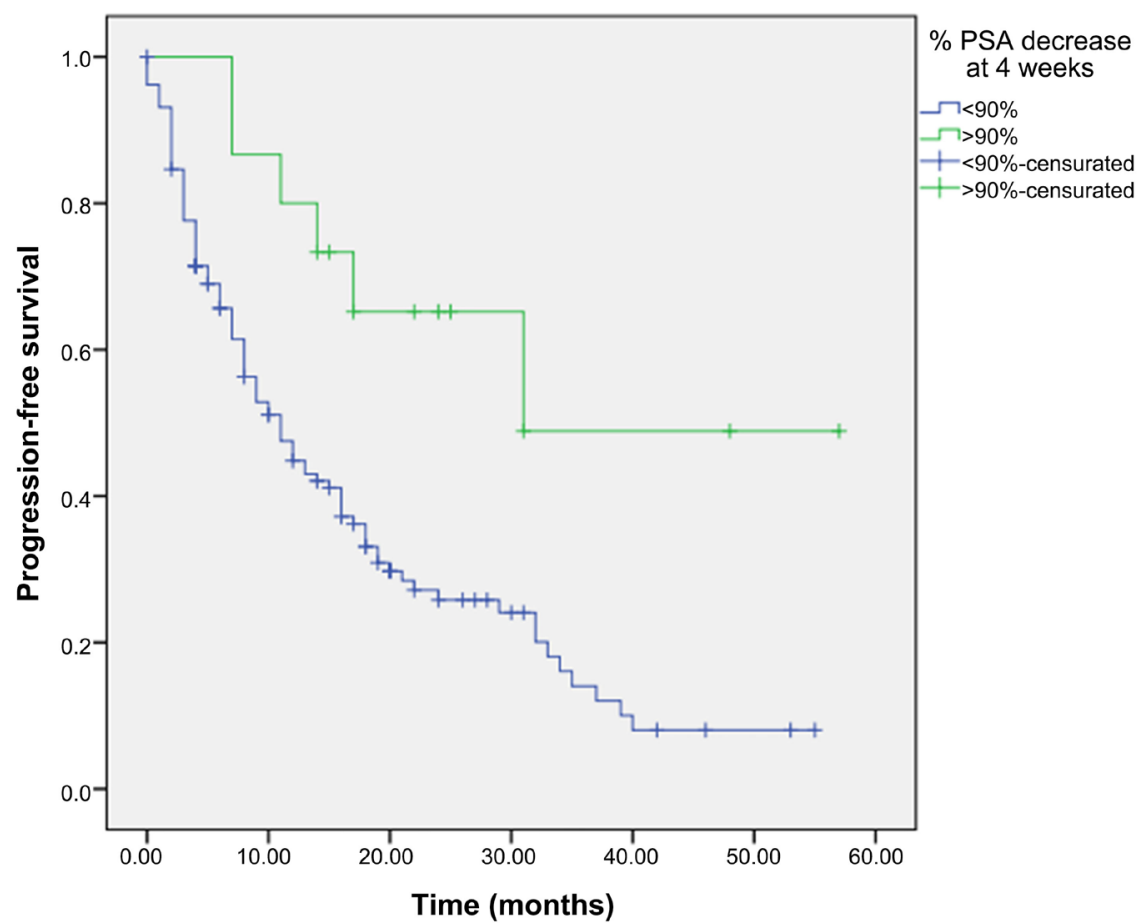

Figure 3. Progression-free survival curve as a function of the decrease in PSA (by $>90 \%$ and $<90 \%)$.

Table 10. Results of survival in m-HSPC group.

\begin{tabular}{cccc}
\hline & $<30 \%$ & $>30 \%$ & $p$ value \\
\hline Median PFS & 3 & 0 & 0.011 \\
Median OS & 5 & 0 & 0.008 \\
\hline Median PFS & $<50 \%$ & $>50 \%$ & $p$ value \\
Median OS & 4 & 0 & 0.146 \\
\hline Mean PFS & 9 & 0 & 0.114 \\
Mean OS & $<90 \%$ & $>90 \%$ & $p$ value \\
\hline
\end{tabular}

Abbreviations: OS, overall survival; PFS, progression-free survival.

Table 11. OS results in the multivariate analysis at 4 weeks.

\begin{tabular}{ccccc}
\hline & Sig. & Exp(B) & \multicolumn{2}{c}{$95.0 \%$ CI for $\operatorname{Exp}(\mathrm{B})$} \\
\cline { 4 - 4 } PSA diagnosis & 0.301 & 1.000 & Upper & Lower \\
Previous CT & 0.001 & 2.764 & 1.000 & 1.001 \\
PSA when AA started & 0.145 & 1.003 & 0.999 & 5.158 \\
Decrease in PSA $>$ 50\% & 0.163 & 1.573 & 0.832 & 1.006 \\
Decrease in PSA $>$ 30\% & 0.95 & 1.02 & 0.55 & 1.91 \\
LDH & 0.581 & 1.000 & 0.999 & 1.001 \\
AP & 0.023 & 1.001 & 1.000 & 1.002 \\
\hline
\end{tabular}

Abbreviations: LDH, low-density lipoprotein; AP, alkaline phosphatase. 
At 8 and 12 weeks, the results were very similar. At 8 weeks, both OS and PFS showed a significant relationship with a PSA decline $>30 \%$ (OS: 33.35 vs. 22.56 $\mathrm{m}, p=0.002$; PFS: 24.78 vs. $10.75 \mathrm{~m}, p<0.001$ ), $>50 \%$ (OS: 37.59 vs. $23.13 \mathrm{~m}, p$ < 0.001; PFS: 25.81 vs. $12.47 \mathrm{~m}, p<0.001$ ) and $>90 \%$ (OS: 28.08 vs. $39.29 \mathrm{~m}, p=$ 0.005 ; PFS: 15.85 vs. $36.51 \mathrm{~m}, p=0.002)$. At 12 weeks, all the survivals found significant relationship with a PSA decline $>30 \%,>50 \%$ and $>90 \%$ as well. However, when the multivariate analysis is performed, these variables lose statistical weigh but a trend is observed.

Regarding the analytical parameters evaluated, we saw a significant decrease in $\mathrm{LDH}$ and AP levels in the periods evaluated in relation to OS and PFS (except for $\mathrm{LDH}$ at 12 weeks). Nonetheless, given that these HRs were remarkably close to 1 , these factors should be considered neutral. The neu/lym index was not statistically significant in any of the time periods we included. Moreover, these parameters were analyzed in each subgroup and the results obtained were similar. Finally, we found a strong correlation between the decrease in PSA at 4 and 8 weeks $($ Rho $=0.912 ; p<0.001), 4$ and 12 weeks $($ Rho $=0.801 ; p<0.001)$, and 8 and 12 weeks $($ Rho $=0.948 ; p<0.001)$.

\section{Discussion}

AA is an effective therapy for metastatic prostate cancer but not without its drawbacks such as some side effects and its cost. The study of the PSA kinetics and its early changes could serve as a tool in daily clinical practice to make a decision about discontinuing the treatment, even earlier than the actual standard.

Our analysis demonstrated a significant improvement in OS and PFS in patients with a decrease in PSA by $\geq 30 \%$ at 4,8 , and 12 weeks. These results coincide with those described in the multicenter study by Rescigno [9] which observed that a decrease in PSA of more than $30 \%$ at 4 weeks was associated with a better OS. Similarly, a decrease in PSA $\geq 50 \%$ was also related to an improved OS and PFS. This confirmed the data previously obtained by Schiff et al. [10] who analyzed PSA levels at 4 and 12 weeks, as well as the results from Facchini [7] who assessed PSA even earlier, 15 days after the start of treatment.

As also found in the post-hoc analysis of the LATITUDE study [11], in our work we observed a correlation between a decrease in PSA by $\geq 90 \%$ and an improvement in PFS. However, we evaluated PSA levels at three specific time points $(4,8$, and 12 weeks) while the analysis in the aforementioned study was carried out when the decrease occurred, rather than at specific points in time. Nonetheless, the relationship between decreased PSA and OS followed the same trend, although it did not reach statistical significance $(p=0.063)$, perhaps because of the limited number of events in our work that achieved this early decrease. Furthermore, an inverse relationship was observed between the tPSAn and survival in both the LATITUDE study and our work, which, although not relevant as a predictive factor because of its retrospective nature, provides valuable retrospective information that can help researchers to better understand the profile of 
non-responders.

Although a decrease in PSA (by $\geq 30 \%$ or $\geq 50 \%$ ) initially correlated with OS in our univariate analysis, the multivariate analysis failed to confirm this. In agreement, the data published by Nakayama [6] showed that a decrease in PSA of more than $30 \%$ at 4 weeks was not a significant predictor of OS. In the univariate analysis, the decrease in PSA by $\geq 30 \%$ or $\geq 50 \%$ was statistically significant for PFS. However, the multivariate analysis only confirmed these decreases as independent prognostic factors when measured at 12 weeks. This finding may be due to several reasons. For example, our sample was heterogeneous compared to the populations studied in the previous literature, which had only included mCRPC or mCPHS patients, but not in the same group. Although we analyzed each patient subgroup separately and did not observe any notable differences between them, this may have been because of the limited sample size in our work. In any case, the data we obtained did not allow us to configure a mathematical predictive model for decreased PSA expression. Nonetheless, it did provide relevant clinical information that could help researchers to individualize the treatment and follow-up of each patient subgroup.

In contrast, our study indicated that the prior administration of chemotherapy constituted an independent risk factor for reduced survival. This finding has already been described elsewhere, such as in the work by Nakayama [6] which observed a difference in PSA response at 4 weeks between patients who had or had not received prior chemotherapy. Perhaps this could be explained by the hypothesis proposed by some authors suggesting that taxanes produce cytoplasmic sequestration of the androgen receptor (AR) and therefore, decrease AR sensitivity which can lead to cross-resistance between docetaxel and AA [14] [15] [16]. Furthermore, it is important to consider that the patient populations who have undergone chemotherapy tend to be older patients who have received numerous previous lines of treatment, a fact that may condition their poorer prognosis.

Other variables that were significant in the univariate analysis and, in some cases also in the multivariate analysis were the LDH and AP levels. However, their impact was doubtful in our study because the HR values were close to 1 , thereby making them neutral variables. Thus, even though these factors have been previously studied and found to be positive prognostic markers, in this current work we could not conclude that they were significant predictive factors.

Another fact worth to mention is that in our study we confirmed the existence of a strong correlation between PSA figures at 4, 8 and 12 weeks, which means that a downward trend is observed from the first 4 weeks and continues until reaching the 12 th week. This is very interesting information for physicians when interpreting the analytical results and being able to predict the most likely future behavior in terms of PSA kinetics.

Finally, we must also assume that our study had several limitations. First, it was a retrospective study. Second, the number of patients included in each subgroup was not as large as would have been desirable. Finally, we were unable to eva- 
luate the PSA flare effect in this current work.

\section{Conclusion}

To conclude, although it cannot be considered an independent predictive variable, an early decrease in PSA could serve as a valuable tool in the early assessment of the effectiveness of second-generation antiandrogens such as AA. This would aid physicians in the timely selection of patients who are unlikely to benefit from this therapy and would thus, help intensify the drive-in current medicine towards individualized therapeutic strategies.

\section{Conflicts of Interest}

The authors have no conflicts of interest to declare.

\section{References}

[1] Ingrosso, G., Detti, B., Scartoni, D., et al. (2018) Current Therapeutic Options in Metastatic Castration-Resistant Prostate Cancer. Seminars in Oncology, 45, 303-315. https://doi.org/10.1053/j.seminoncol.2018.10.001

[2] Ritch, C. and Cookson, M. (2018) Recent Trends in the Management of Advanced Prostate Cancer [Version 1; Peer Review: 3 Approved]. F1000Research, 7, 1513. https://doi.org/10.12688/f1000research.15382.1

[3] De Bono, J. and Logothetis, C. (2011) Abiraterone and Increased Survival in Metastatic Prostate Cancer. The New England Journal of Medicine, 364, 1995-2005. https://doi.org/10.1056/NEJMoa1014618

[4] Ryan, C., Smith, M., De Bono, J., Molina, A., Logothetis, C. and De Souza, P. (2013) Abiraterone in Metastatic Prostate Cancer without Previous Chemotherapy. The New England Journal of Medicine, 368, 138-148. https://doi.org/10.1056/NEJMoa1209096

[5] Scher, H.I., Morris, M.J., Stadler, W.M., et al. (2016) Trial Design and Objectives for Castration-Resistant Prostate Cancer: Updated Recommendations from the Prostate Cancer Clinical Trials Working Group 3. Journal of Clinical Oncology, 34, 1402-1418. https://doi.org/10.1200/JCO.2015.64.2702

[6] Nakayama, M., Kobayashi, H., Takahara, T., Oyama, R., Imanaka, K. and Yoshizawa, K. (2016) Association of Early PSA Decline and Time to PSA Progression in Abiraterone Acetate-Treated Metastatic Castration-Resistant Prostate Cancer; a PostHoc Analysis of Japanese Phase 2 Trials. BMC Urology, 16, 1-8. https://doi.org/10.1186/s12894-016-0148-4

[7] Facchini, G., Caffo, O., Ortega, C., et al. (2016) Very Early PSA Response to Abiraterone in mCRPC Patients: A Novel Prognostic Factor Predicting Overall Survival. Frontiers in Pharmacology, 7, 123. https://doi.org/10.3389/fphar.2016.00123

[8] Schlack, K., Krabbe, L.M., Fobker, M., Schrader, A.J., Semjonow, A. and Boegemann, M. (2016) Early Prediction of Therapy Response to Abiraterone Acetate Using PSA Subforms in Patients with Castration Resistant Prostate Cancer. International Journal of Molecular Sciences, 17, 1520. https://doi.org/10.3390/ijms17091520

[9] Rescigno, P., Lorente, D., Bianchini, D., et al. (2016) Prostate-Specific Antigen Decline after 4 Weeks of Treatment with Abiraterone Acetate and Overall Survival in Patients with Metastatic Castration-Resistant Prostate Cancer. European Urology, 
70, 724-731. https://doi.org/10.1016/j.eururo.2016.02.055

[10] Schiff, J.P., Cotogno, P., Feibus, A., et al. (2019) Early Prostate-Specific Antigen Response Post-Abiraterone as Predictor of Overall Survival in Metastatic Castrate-Resistant Prostate Cancer. BMC Cancer, 19, Article No. 524.

https://doi.org/10.1186/s12885-019-5729-7

[11] Matsubara, N., Chi, K.N., Özgüroğlu, M., et al. (2020) Correlation of Prostate-Specific Antigen Kinetics with Overall Survival and Radiological Progression-Free Survival in Metastatic Castration-Sensitive Prostate Cancer Treated with Abiraterone Acetate plus Prednisone or Placebos Added to Androgen Deprivatio. European Urology, 77, 494-500. https://doi.org/10.1016/j.eururo.2019.11.021

[12] Sheng, I.Y., Fallah, J., Gupta, R., et al. (2020) Identifying Prostate Surface Antigen Patterns of Change in Patients with Metastatic Hormone Sensitive Prostate Cancer Treated with Abiraterone and Prednisone. Targeted Oncology, 15, 477-483. https://doi.org/10.1007/s11523-020-00734-w

[13] UICC-Union for International Cancer Control. https://www.uicc.org

[14] Loriot, Y., Bianchini, D., Ileana, E., et al. (2013) Antitumour Activity of Abiraterone Acetate against Metastatic Castration-Resistant Prostate Cancer Progressing after Docetaxel and Enzalutamide (MDV3100). Annals of Oncology, 24, 1807-1812. https://doi.org/10.1093/annonc/mdt136

[15] Mezynski, J., Pezaro, C., Bianchini, D., et al. (2012) Antitumour Activity of Docetaxel Following Treatment with the CYP17A1 Inhibitor Abiraterone: Clinical Evidence for Cross-Resistance? Annals of Oncology, 23, 2943-2947.

https://doi.org/10.1093/annonc/mds119

[16] Zhu, M.L., Horbinski, C.M., Garzotto, M., Qian, D.Z., Beer, T.M. and Kyprianou, N. (2010) Tubulin-targeting Chemotherapy Impairs Androgen Receptor Activity in Prostate Cancer. Cancer Research, 70, 7992-8002. https://doi.org/10.1158/0008-5472.CAN-10-0585 